\title{
Radiationless Relaxation in a Synthetic Analogue of the Green Fluorescent Protein Chromophore
}

\author{
Naomi M. Webber, Konstantin L. Litvinenko, and Stephen R. Meech* \\ School of Chemical Sciences, University of East Anglia, Norwich NR4 7TJ, U.K.
}

Received: April 17, 2001; In Final Form: June 1, 2001

\begin{abstract}
The fluorescence and ultrafast ground-state recovery times of the isolated chromophore of the green fluorescent protein have been studied in basic alcohol solutions. The fluorescence quantum yield increases more than $10^{3}$ times between 295 and $77 \mathrm{~K}$. The major part of the increase occurs in the supercooled liquid range, and continues below the glass transition. The ground-state recovery at $295 \mathrm{~K}$ is essentially (95\%) complete in under $5 \mathrm{ps}$, is nonexponential, and only weakly dependent on solvent viscosity. These results are inconsistent with a viscosity-controlled radiationless process involving large scale intramolecular reorganization. If intramolecular motion is involved it must be of small scale. Alternative mechanisms are discussed. A thermally activated radiationless decay process is consistent with the present data, but the mechanism is unclear. For either mechanism the high quantum yield in the intact protein must arise through protein-chromophore interactions which effectively suppress the radiationless channel.
\end{abstract}

\section{Introduction}

The green fluorescent protein (GFP) is established as a highly useful tool in fluorescence studies of living cells. ${ }^{1}$ Its electronic spectra and fluorescence intensity are strong functions of protein mutation and environment, suggesting that the protein may be engineered for specific applications. ${ }^{1,2}$ To properly pursue this objective a detailed understanding of the photophysics of the GFP chromophore is required. Studies of intact GFP suggest that its photophysical behavior is not straightforward: the native protein has a fluorescence quantum yield, $\Phi_{\mathrm{f}}$, of $0.8,{ }^{3}$ while the isolated chromophore in solution has $\Phi_{\mathrm{f}}<10^{-3.4}$ the protein switches between dark and bright states under illumination; ${ }^{5}$ the fluorescence is sensitive to $\mathrm{pH}^{6}$

In an attempt to provide a detailed picture of the photophysics of GFP we have synthesized model compounds of the chromophore responsible for the fluorescence. Here we report a preliminary investigation of the ultrafast relaxation dynamics and temperature-dependent fluorescence of the isolated chromophore of GFP. It is found that at $295 \mathrm{~K}$ the dominant part of the ground-state recovery occurs with sub picosecond to picosecond time constants, which are only weakly dependent on medium viscosity, $\eta$. The fluorescence intensity increases dramatically with decreasing temperature, with the main part of the increase occurring in the supercooled liquid, and continuing below the glass transition. These findings are not consistent with the proposition that radiationless relaxation in the isolated chromophore (and nonfluorescent mutants of GFP) occurs through large scale torsional motion in the excited state. ${ }^{7}$ Instead the data suggest that the protein matrix has an influence on the excited state of the chromophore that is more subtle than simple restriction of intramolecular motion.

\section{Experimental Section}

The chromophore ( $p$-hydroxybenzylidene-imidazolidinone, I) was synthesized according to the method of Kojima et al. ${ }^{8}$ It

* Author to whom correspondence should be addressed. E-mail: s.meech@ uea.ac.uk.

\section{SCHEME 1}<smiles>CC1=N/C(=C\c2ccc([O-])cc2)C(=O)N1C</smiles>

was dissolved in alcohol solvents to which $1 \% 1 \mathrm{M} \mathrm{NaOH}$ was added, to generate the anion. Formation of the anion was observed through absorption spectroscopy, as a $70 \mathrm{~nm}$ shift of the $S_{0}-S_{1}$ transition to longer wavelength. This form of $\mathbf{I}$ is most similar to the fluorescent state of GFP, ${ }^{4}$ although the transition in the protein is shifted to still lower energy. Fluorescence spectra were measured between 302 and $77 \mathrm{~K}$. Ultrafast polarization spectroscopy was employed to measure the ground-state recovery kinetics of $\mathbf{I}$ in basic alcohol solutions of different viscosity at $295 \mathrm{~K}$ with 100 fs time resolution. ${ }^{9}$ The ultrafast apparatus has been described elsewhere. ${ }^{10}$ For the data reported here the lowest electronic absorption band of $\mathbf{I}$ was excited by a linearly polarized pulse at $396 \mathrm{~nm}$. The groundstate recovery was probed at the same wavelength by a time delayed pulse polarized at $45^{\circ}$ to the excitation pulse. The probe intensity was measured behind a polarizer oriented at $-43.5^{\circ}$ to the excitation pulse. In this heterodyned detection geometry the sample dichroism is measured with excellent signal-to-noise, and the dynamics contain information on both population relaxation and orientational dynamics. ${ }^{9,11}$ On the ultrafast time scale $(<10 \mathrm{ps})$ population relaxation is assumed to dominate.

\section{Results and Discussion}

In Figure 1a the fluorescence spectra in basic ethanol are plotted as a function of temperature. Two factors deserve comment. First, the intensity increases by a factor of $10^{3}-10^{4}$ between 295 and $77 \mathrm{~K}$, consistent with previous reports that $\Phi_{\mathrm{f}}<10^{-3}$ at room temperature. ${ }^{4}$ Second, there is also a small shift $\left(900 \mathrm{~cm}^{-1}\right.$, difficult to discern in Figure 1) of the 

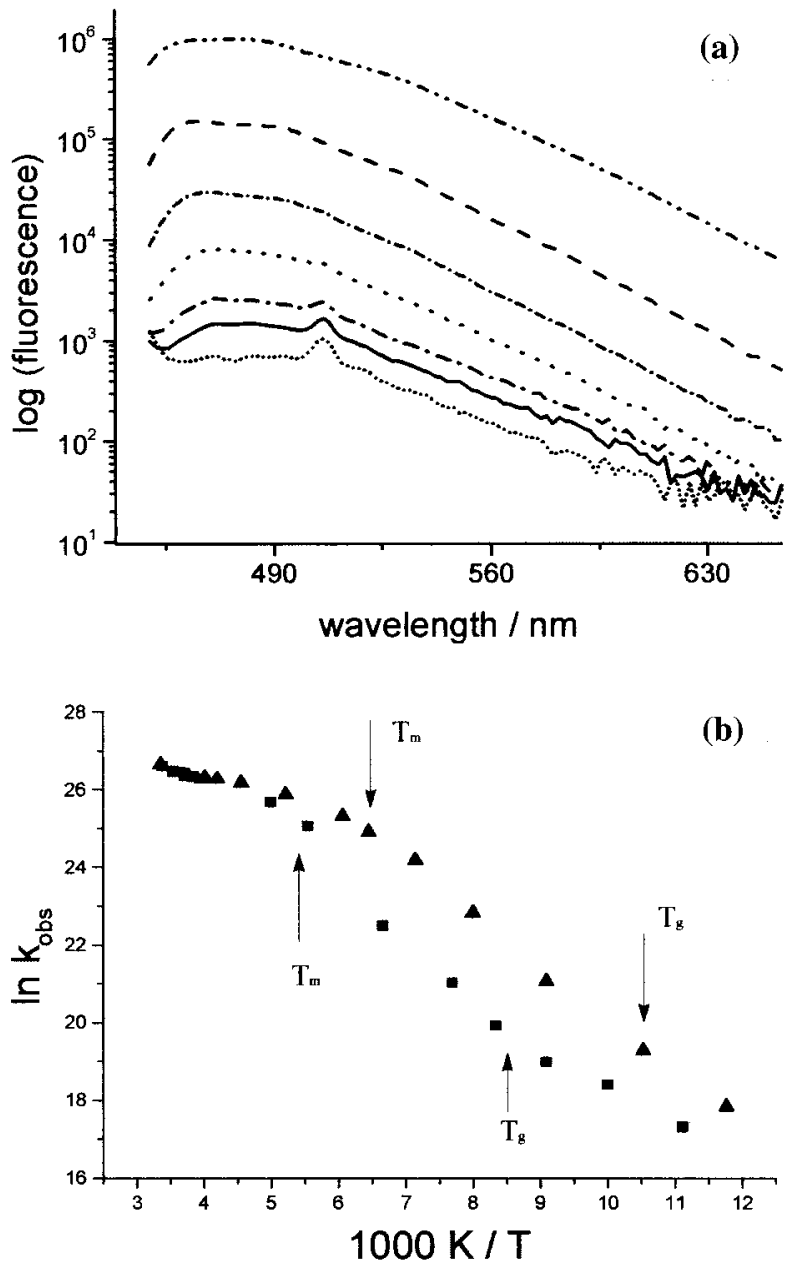

Figure 1. (a) Fluorescence spectra for $\mathbf{I}$ in basic ethanol (log scale), increasing with decreasing temperature, from bottom $302 \mathrm{~K}, 192 \mathrm{~K}$, $165 \mathrm{~K}, 140 \mathrm{~K}, 125 \mathrm{~K}, 110 \mathrm{~K}, 77 \mathrm{~K}$. The excitation wavelength was $440 \mathrm{~nm}$. The feature at $509 \mathrm{~nm}$ is solvent Raman. (b) An Arrhenius plot for $k_{\mathrm{obs}}$ of I measured in basic ethanol (triangles) and butanol (squares) solutions. The melting and glass transition temperatures are marked.

fluorescence to higher energy with decreasing temperature. The direction of the shift is consistent with the freezing out of excited-state solvation dynamics about the changed dipole moment of the excited state. ${ }^{12}$ Bublitz et al. ${ }^{13}$ reported the change in dipole moment on excitation of GFP as $6.8 \mathrm{D}$, consistent with the observation of a spectral shift. The shift observed is smaller than expected from established models of solvation, ${ }^{12}$ which may be associated with the very short lifetime of the excited state of $\mathbf{I}$ in ethanol at $295 \mathrm{~K}$ (see below).

The temperature-dependent radiationless rate, $k_{\mathrm{obs}}$, was calculated according to $k_{\mathrm{obs}}=k_{f}\left[\left\{\left(1-\Phi_{\mathrm{f}}\right)\right\} /\left\{\Phi_{\mathrm{f}}\right\}\right],{ }^{14}$ where $k_{\mathrm{f}}$ is the radiative decay rate (assumed, on the basis of the fluorescence lifetime of GFP, ${ }^{15}$ to be equal to $0.25 \times 10^{9} \mathrm{~s}^{-1}$ ), and $\Phi_{\mathrm{f}}$ is the quantum yield, measured relative to the value at $77 \mathrm{~K}$. The natural logarithm of $k_{\mathrm{obs}}$ is plotted as a function of the reciprocal temperature for ethanol and butanol solutions in Figure 1b. The plot is approximately linear between 77 and 170 $\mathrm{K}$, with slopes, expressed as Arrhenius activation energies, of $11 \mathrm{~kJ} \mathrm{~mol}^{-1}$ for both ethanol and butanol. There is an intriguing break in the plot for butanol (but not ethanol) at the glass transition temperature. This might indicate a sensitivity of $k_{\mathrm{obs}}$ to the glass transition, but the effect is small. The slope also decreases above the melting temperature (to $E_{\mathrm{a}}=3.8$ and 4.6 $\mathrm{kJ} \mathrm{mol}^{-1}$ for ethanol and butanol, respectively). In the context

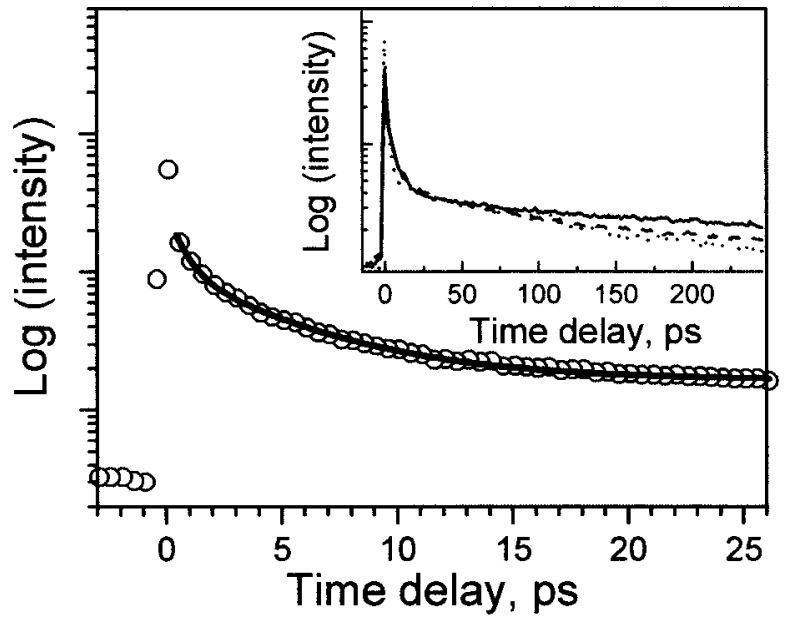

Figure 2. The ultrafast polarization spectroscopy measurement for I in basic butanol, fit to 2 exponential relaxation terms (plus $\tau_{\text {long }}$ ). The inset shows the long time relaxation for different solvents (solid line: butanol; dashed line: ethanol; dotted line: water).

of the radiationless decay, the most interesting feature of Figure $1 b$ is that the major part of the increase in $\Phi_{\mathrm{f}}$ occurs in the supercooled liquid and glass regions, i.e., the sample is weakly fluorescent at $170 \mathrm{~K}$, but strongly so at $77 \mathrm{~K}$ (Figure 1). These data alone are not sufficient to assign $k_{\mathrm{obs}}$ to a temperatureactivated mechanism, since viscosity is also a function of temperature. However, we note that for propanol (where data are available, ethanol and butanol being assumed to behave in an analogous fashion) the viscosity increases from $2 \mathrm{cP}$ at 295 $\mathrm{K}$ to $1.9 \times 10^{6} \mathrm{cP}$ at $T_{\mathrm{g}}+26 \mathrm{~K}$, and finally to $>10^{10} \mathrm{cP}$ at $T_{\mathrm{g}}$ $+4 \mathrm{~K} ;{ }^{16}$ if the radiationless mechanism is viscosity dependent it is not a linear relationship.

To further characterize the radiationless mechanism, ultrafast transient dichroism measurements of the ground-state recovery times were made in alcohol solvents of different viscosity at $295 \mathrm{~K}$. Although parameters other than viscosity change when the sample is varied between methanol and butanol (polarity, H-strength) it was observed that the absorption spectra of I were nearly independent of solvent (spectral shift of $<2 \mathrm{~nm}$ ). The transient data are shown in Figure 2, for intermediate and long time scales (inset). Measurements on a faster time scale (0-2 ps, not shown) show only a time zero "coherence spike" and smooth exponential relaxation.

The data of Figure 2 reveal a dominant (>90\%) ultrafast relaxation and a minor, slower relaxation. The fast relaxation is assigned to ultrafast ground-state recovery (GSR) through internal conversion (IC) followed by vibrational relaxation (VR) in the ground state. The picosecond GSR time places an upper limit on the excited-state decay time, consistent with the observed fluorescence quantum yield of $<10^{-3}$. ${ }^{4}$ The ultrafast GSR is nonexponential, and is fit by two components. The important result with regard to mechanism is that the GSR times are nearly independent of solvent viscosity (Table 1).

The observation of an additional slower relaxation suggests a minor channel in the GSR cycle leading to a bottleneck. The slow viscosity-dependent relaxation seen in Figure 2 (inset) may then be ascribed to orientational relaxation. These slow dynamics are currently the subject of more detailed analysis. ${ }^{17}$

A plausible mechanism for the observed ultrafast IC in $\mathbf{I}$ is torsional motion in the excited state. Torsional motion may cause ground and excited states to approach in energy, or cross, dramatically enhancing the rate of IC. ${ }^{18}$ Such a mechanism has already been proposed for some of the weakly fluorescent 
TABLE 1: Parameters for Exponential Fits to Transient Data in Three Solvents (all containing $1 \%$ base) ${ }^{a}$

\begin{tabular}{lccc}
\hline & butanol & ethanol & methanol \\
\hline$\eta(\mathrm{cP})$ & 2.6 & 1.1 & 0.5 \\
$<\tau>(\mathrm{ps})$ & 2.0 & 1.3 & 2.4 \\
$\tau_{\text {long }}(\mathrm{ps})$ & 288 & 152 & 93
\end{tabular}

${ }^{a}$ Data were recovered from a triexponential fit starting at $t=1 \mathrm{ps}$. The long relaxation time was held fixed at a value determined in separate experiments. The mean lifetime $\langle\tau\rangle$ is the average of the two fast relaxation times weighted by their pre exponents

mutants of GFP. ${ }^{7,19}$ The time scale of the IC observed here is as fast, or faster, than expected for such torsional motion in solution (for example, in the well characterized barrierless torsion in crystal violet, IC occurs in 9 ps in butanol ${ }^{20}$ ). Candidates for the torsional motion in the excited state of $\mathbf{I}$ were considered in the quantum chemical calculations of Weber et al.. ${ }^{21}$ Ground and excited-state potential energies were calculated for $0^{\circ}$ and $90^{\circ}$ twisted conformers of three potential torsional modes. Although there are differences between the three cases, the excited and ground states were observed to approach, as required for fast IC. Two of these torsional motions involve twisting around individual carbon-carbon bridging bonds. Such intramolecular rotation in I would require the displacement of a considerable volume of solvent. Such motion will be opposed by solvent friction. In the case of large scale intramolecular motion the high friction, diffusive, limit will apply, and the GSR time is expected to scale linearly with viscosity. ${ }^{20}$ This dependence is clearly not observed for $\mathbf{I}$, the observed ultrafast GSR times are essentially independent of viscosity (Table 1).

The weak viscosity dependence of the ultrafast GSR at 295 $\mathrm{K}$ (Figure 2) and the continuous decrease of $k_{\mathrm{obs}}$ through the extremely viscous, supercooled liquid phase, and into the glassy state (Figure 1) both argue against a radiationless mechanism controlled by bulk viscosity. This seems to rule out IC induced by internal rotation of the aromatic rings about either of the two carbon-carbon bridging bonds. The third isomerization pathway considered in the calculations of Weber et al. ${ }^{21}$ was a concerted rotation of both the bridging bonds, leaving the aromatic rings in place (the "hula twist"). This would require the displacement of a small solvent volume, and hence be less likely to be controlled by macroscopic viscosity. It is plausible that such a small scale intramolecular motion persists even in the glassy state. There are precedents for torsional motion being observed below the melting and glass transition temperatures, in particular Ye et al. observed that the fluorescence lifetime of malachite green continued to increase below $T_{m}$ and $T_{g}{ }^{22}$ The excited state torsion believed to be active in the IC of malachite green is an approximately $10^{\circ}$ rotation of the phenyl rings. ${ }^{20}$ It was suggested that this motion was not fully coupled to the macrosopic medium viscosity. There are similarities between the data of Figure $1 \mathrm{~b}$ and that of Ye et al., in that they also observed a discontinuity in the plot in the region $50 \mathrm{~K}$ above $T_{\mathrm{g}}$, similar to that seen in Figure $1 \mathrm{~b}$, around $T_{\mathrm{m}}$. However, there are important differences as well, in that Ye et al. report a significant second discontinuity at $T_{\mathrm{g}}$, which is absent for ethanol and, at most, only barely discernible in the butanol data. In addition the excited-state decay time of malachite green at room temperature ${ }^{20}$ is much more sensitive to viscosity than was observed for I (Table 1). We conclude that the present data may be consistent with IC induced by excited-state torsional motion only if the motion involves the displacement of very small solvent volumes. The "hula twist" is the only obvious candidate. Thus, this conclusion does not rule out the possibility

\section{SCHEME 2}

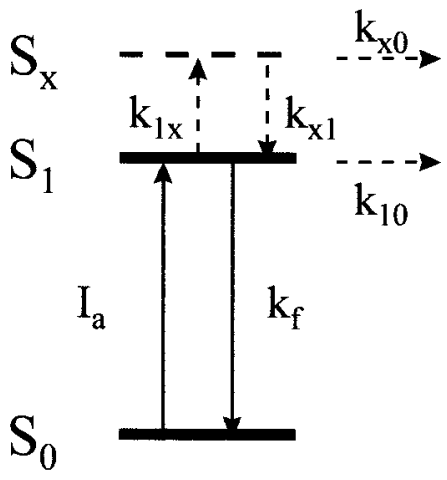

of an effect of medium viscosity on the radiationless rate. It does suggest that the effect is weak, being significant only at very high viscosity. We are currently studying the photophysics of $\mathbf{I}$ in a range of glass-forming solvents, including glycerol and propylene glycol.

An alternative to a viscosity-controlled (however weakly) IC mechanism is a thermally activated one, in which a state $S_{x}$, located $1100 \mathrm{~cm}^{-1}$ above the thermally relaxed level of $S_{1}$, is strongly coupled to the ground state. This is represented in the kinetic scheme shown in Scheme 2, which yields, in steady state, $k_{\mathrm{obs}}=\left\{k_{1 x} k_{x 0}\right\} /\left\{\left(k_{x 1}+k_{x o}\right)\right\}$, which reduces to $k_{\mathrm{obs}}=k_{x 0}$ exp$\left(\left\{-\Delta G_{1 x}\right\} /\{R T\}\right)$ under the assumption of a rapid thermal equilibrium between $\mathrm{S}_{x}$ and $\mathrm{S}_{1}$. This is consistent with the approximately Arrhenius behavior seen in the low temperature region (Figure 1b). The difficulty with the thermally activated mechanism is the identity of the state $S_{x}$. One possibility is a vibronic level of $S_{1}$ coupled to a point of intersection of the $S_{0}$ and $S_{1}$ states. Such conical intersections have been identified in several molecules, ${ }^{23}$ and may be accessed without large scale intramolecular reorganization, the classical example being the rapid IC in azulene. ${ }^{23}$ A second possibility is that $S_{x}$ is a second excited state. There are reported cases where thermal population of an $S_{2}$ state of $n \pi^{*}$ character, coupled to both $S_{1}\left(\pi \pi^{*}\right)$ and $S_{0}$ results in rapid IC. ${ }^{24}$ However, it should be noted that no such state has been identified in the available molecular orbital calculations on $\mathbf{I} .{ }^{25}$ The latter mechanism is expected to yield a solvent dependence of $\Phi_{\mathrm{f}}$ as solvent solute interactions shift the $\mathrm{n} \pi^{*}-\pi \pi^{*}$ energy gap. ${ }^{24}$ In studies of the neutral form of $\mathbf{I}$ at room temperature no large solvent effect has been observed. ${ }^{17}$

\section{Conclusion}

The fluorescence and ultrafast ground-state recovery dynamics of a model GFP chromophore have been studied. The model compound is only very weakly fluorescent at $295 \mathrm{~K}$. The mechanism of nonradiative decay is IC/VR. Ultrafast transient dichroism measurements show that the rate of relaxation at room temperature is nearly independent of viscosity. The fluorescence quantum yield increases dramatically with decreasing temperature, particularly in the supercooled liquid and glass phase. These data are not consistent with an IC mechanism involving large scale intramolecular reorganization. Small scale intramolecular reorganization leading to a crossing of $S_{1}$ and $S_{0}$ is one possible mechanism, and the "hula twist" motion described by Weber et al. is a possibility. Additional thermally activated IC mechanisms have been discussed. Internal conversion arising from a thermally populated level above $S_{1}$ is consistent with most of the data. However, there is insufficient data in the available quantum chemical calculations to assign (or rule out) these mechanisms. Collectively, the data are consistent with IC 
at an $\mathrm{S}_{0} / \mathrm{S}_{1}$ intersection accessed via an energy barrier in a coordinate that experiences only weak solvent frictional forces.

The significance of these results for GFP and its mutants is that the protein is evidently acting as more than a viscous cage restricting torsional motion of the chromophore. Specific interactions between the protein and the chromophore appear to have the ability to "turn off" the radiationless channel. Further work to identify both the radiationless mechanism in $\mathbf{I}$ and the nature of the protein pigment interactions is in progress.

Acknowledgment. We are grateful to Dr. G. R. Stephenson's group (UEA) for assistance with the synthesis of $\mathbf{I}$, and EPSRC and BBSRC for financial support. We are also grateful to the referees for their helpful comments. N.W. thanks EPSRC for a studentship.

\section{References and Notes}

(1) Tsien, R. Y. Annu. Rev. Biochem. 1998, 67, 509.

(2) Heim, R.; Tsien, R. Y. Curr. Biol. 1996, 6, 178.

(3) Morise, H.; Shimomura, O.; Johnson, F. H.; Winant, J. Biochemistry 1974, 13, 2656.

(4) Niwa, H.; Inouye, S.; Hirano, T.; Matsuno, T.; Kojima, S.; Kubota, S.; Ohashi, M.; Tsuji, F. I. Proc. Natl. Acad. Sci. U.S.A. 1996, 93, 13617.

(5) Schwille, P.; Kummer, S.; Heikal, A. A.; Moerner, W. E.; Webb, W. W. Proc. Natl. Acad. Sci. U.S.A. 2000, 97, 151.

(6) Elsliger, M.-A.; Wachter, R. M.; Hanson, G. T.; Kallio, K.; Remington, S. J. Biochemistry 1999, 38, 5296.

(7) Voityuk, A. A.; Michel-Beyerle, M.-E.; Rösch, N. Chem. Phys. Lett. 1998, 296, 269.

(8) Kojima, S.; Ohkawa, H.; Hirano, T.; Maki, S.; Niwa, H.; Ohashi, M.; Inouye, S.; Tsuji, F. I. Tetrahedron Lett. 1998, 39, 5239.
(9) Cross, A. J.; Waldeck, D. H.; Fleming, G. R. J. Chem. Phys. 1983, 78,6455 .

(10) Lin, S.; Hands, I. D.; Andrews, D. L.; Meech, S. R. J. Phys. Chem. A 1999, 103, 3830; Smith, N. A.; Meech, S. R. J. Phys. Chem. A 2000, $104,4223$.

(11) Alavi, D. S.; Hartman, R. S.; Waldeck, D. H. J. Chem. Phys. 1990, 92, 4055 .

(12) Maroncelli, M. J. Mol. Liq. 1993, 57, 1.

(13) Bublitz, G.; King, B. A.; Boxer, S. G. J. Am. Chem. Soc. 1998 120,9370 .

(14) Sun, Y.; Saltiel, J. J. Phys. Chem. 1989, 93, 8310.

(15) Perozzo, M. A.; Ward, K. B.; Thomson, R. B.; Ward, W. W. J. Biol. Chem. 1988, 263, 7716.

(16) Ling, A. C.; Willard, J. E. J. Phys. Chem. 1968, 72, 1918.

(17) Litvinenko, K.; Webber, N.; Meech, S. R. In preparation.

(18) Ben-Amotz, D.; Harris, C. B. Chem. Phys. Lett. 1985, 119, 305, and references therein.

(19) Kummer, A. D.; Wiehler, J.; Rehaber, H.; Kompa, K.; Steipe, B.; Michel-Beyerle, M. E. J. Phys. Chem. B 2000, 104, 4791; Kummer, A. D.; Kompa, K.; Lossau, H.; Pöllinger-Dammer, F.; Michel-Beyerle, M. E.; Silva, C. M.; Bylina, E. J.; Coleman, W. J.; Yang, M. M.; Youvan, D. C. Chem. Phys. 1998, 237, 183 .

(20) Ben-Amotz, D.; Harris, C. B. J. Chem. Phys. 1987, 86, 4856.

(21) Weber, W.; Helms, V.; Mccammon, J. A.; Langhoff, P. W. Proc. Natl. Acad. Sci. U.S.A. 1999, 96, 6177.

(22) Ye, J. Y.; Hattori, T.; Inouye, H.; Ueta, H.; Nakatsuka, H.; Maruyama, Y.; Ishikawa, M. Phys. Rev. B 1996, 53, 8349.

(23) Bearpark, M. J.; Bernardi, F.; Clifford. S.; Olivucci, M.; Robb, M. A.; Smith, B. R. J. Am. Chem. Soc. 1996, 118, 5254; Bearpark, M. J.; Bernardi, F.; Olivucci, M.; Robb, M. A.; Smith, B. R.,; Vreven, T. J. Am. Chem. Soc. 1996, 118, 169.

(24) De Melo, J. S.; Becker, R. S.; Elisei, F.; Maçanita, A. L. J. Chem. Phys. 1997, 107, 6062.

(25) Voityuk, A. A.; Michel-Beyerle, M.-A.; Rösch, N. Chem. Phys. 1998, 231, 13. 